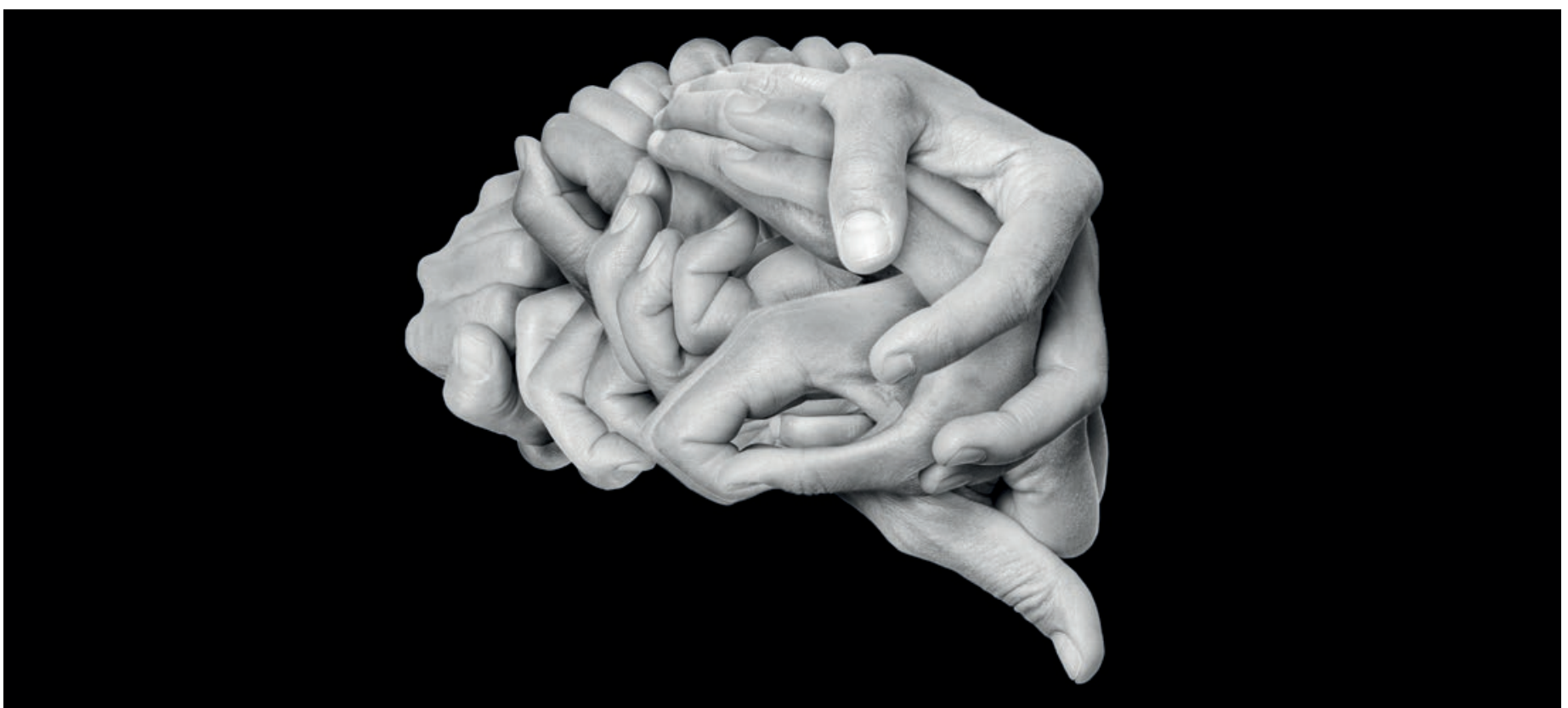

Die Women in Neurology vernetzen sich, um Ärztinnen zu fördern

\title{
Women in Neurology: Förderung der Chancengleichheit
}

\section{Barbara Tettenborn ${ }^{a}$, Andrea Humm ${ }^{b}$}

a Prof. Dr. med., Präsidentin der "Women in Neurology», Kantonsspital St. Gallen, St. Gallen;

b PD Dr. med., Beisitzerin im Vorstand der Schweizerischen Neurologischen Gesellschaft (SNG), HFR Freiburg - Kantonsspital, Freiburg

Die Women in Neurology, kurz WIN, sind Teil der Schweizerischen Neurologischen Gesellschaft. Sie wollen Karrieremöglichkeiten für Ärztinnen fördern und setzen dabei zum Beispiel auf Mentoringprogramme und Vernetzung.

Seit mehreren Jahren sind es vor allem Frauen, die Medizin studieren und sich zu Fachärztinnen für Neurologie weiterbilden. Dennoch sind Frauen in der Neurologie unter den Kaderärztinnen und -ärzten, den Leitenden Ärztinnen und Ärzten sowie den Chefärztinnen und -ärzten noch immer in der Minderheit und stark unterrepräsentiert.

So hat eine kürzlich durchgeführte Umfrage unter den neurologischen Weiterbildungsstätten der Kategorie A ergeben, dass 70 Prozent der Assistenzärztinnen und -ärzte weiblich sind. Bei den Oberärztinnen und -ärzten sinkt der Frauenanteil dann auf 50 Prozent, und von den Chefärztinnen und -ärzten sowie den Leitenden Ärztinnen und Ärzten sind weniger als 20 Prozent weiblich
Aus diesem Grund hat eine Gruppe von erfahrenen, in der Schweizerischen Neurologischen Gesellschaft (SNG) engagierten Neurologinnen aus der ganzen Schweiz am 12. Februar 2020 in Olten eine Gruppe eigens für Assistenzärztinnen sowie Fachärztinnen für Neurologie ins Leben gerufen: die Women in Neurology, kurz WIN.

\section{Mentoring-Programm für Ärztinnen geplant}

Die WIN vertreten als Vereinigung der SNG die Interessen aller Assistenzärztinnen und Fachärztinnen in der Neurologie. Es ist das zentrale Anliegen des Gremiums, die Chancengleichheit und die Karrieremöglichkeiten 


\section{Der Vorstand der Women in Neurology} (WIN)

Prof. Dr. med. Barbara Tettenborn, Präsidentin PD Dr. med. Susanne Renaud, Vizepräsidentin Prof. Dr. med. Margitta Seeck, Academic Development PD Dr. med. Mira Katan Kahles, Coaching/Mentoring Prof. Dr. med. Susanne Wegener, Coaching/Mentoring PD Dr. med. Andrea Humm, Vertretung SNG Dr. med. Karin Ackermann, Vertretung SGKN PD Dr. med. Marie-Luise Mono, Vertretung SHG Prof. Dr. med. Caroline Pot Kreis, Vertretung MS-Gesellschaft pract.med. Noëlle Mercier, Vertretung Schweizerische EpilepsieLiga

für Frauen im Fachgebiet der Neurologie zu unterstützen und zu verbessern. Die Vereinigung will es Assistenzärztinnen und Fachärztinnen bereits zu einem frühen Zeitpunkt in der Karriere ermöglichen, mit Kolleginnen aus der ganzen Schweiz in Kontakt zu treten sowie Erfahrungen und Ideen auszutauschen.

Die WIN wollen eine niederschwellige Anlaufstelle für "Karrierefragen» sein. Aktuell wird deshalb unter anderem ein Mentoring-Programm erarbeitet, das den Wissens- und Erfahrungsaustausch zwischen erfahrenen Neurologinnen in leitenden Positionen und jungen Oberärztinnen sowie Assistenzärztinnen auf persönlicher Ebene ermöglichen soll.

\section{Vernetzung mit anderen Organisationen}

Ebenso wichtig wie der interne Austausch unter den Mitgliedern der WIN ist der Kontakt zu anderen Organisationen und Gremien innerhalb der Neurologie und der klinischen Neurowissenschaften. Ein weibliches Vorstandsmitglied der SNG ist jeweils auch Mitglied des WIN-Vorstandes, um die enge Beziehung zwischen dem SNG-Vorstand und der Vereinigung zu garantieren.
Ausserdem soll wenn immer möglich ein Mitglied des WIN-Vorstandes in der Schweizerischen Gesellschaft für klinische Neurophysiologie (SGKN), in der Schweizerischen Hirnschlag Gesellschaft (SHG), in der Schweizerischen Multiple Sklerose Gesellschaft und in der Schweizerischen Epilepsie-Liga im Vorstand vertreten sein. Die WIN prüfen im weiteren Verlauf auch ein Engagement im Netzwerk der Swiss Federation of Clinical Neuro-Societies (SFCNS), der insgesamt 14 Mitgliedergesellschaften aus den klinischen Neurodisziplinen angehören. Mittelfristiges Ziel ist es, eine einheitliche Stimme über die Grenzen der einzelnen Neurodisziplinen hinweg zu bilden. An den Jahrestagungen der SNG organisieren die WIN jeweils ein Forum, um wichtige Angelegenheiten zu diskutieren und sich für die $\mathrm{Zu}$ kunft und ihre Herausforderungen zu positionieren. Die Präsentation der WIN und ihrer Aktivitäten auf der Homepage der SNG ist derzeit noch im Aufbau.

Symbolbild

Angelo Cordeschi|Dreamstime.com

\section{Das Wichtigste in Kürze}

- 70 Prozent der Assistenzärztinnen und -ärzte sind weiblich, aber weniger als 20 Prozent der Chefärztinnen und -ärzte sowie der Leitenden Ärztinnen und Ärzte.

logische Gesellschaft SNG c/o IMK Institut für Medizin und Kommunikation AG

Münsterberg 1

CH-4001 Basel swissneuro[at]imk.ch www.swissneuro.ch
Die Women in Neurology wollen ein niederschwelliges Angebot für Karrierefragen bieten.

- Mit Hilfe eines Mentoring-Programms und durch gute Vernetzung mit verschiedenen Fachgesellschaften sollen Frauen unterstützt werden. 